\title{
Partial wave decomposition of neutron matter equation of state
}

\section{Davesne and P. Becker}

Université de Lyon, F-69003 Lyon, France; Université Lyon 1, 43 Bd. du 11 Novembre 1918, F-69622 Villeurbanne cedex, France

\section{A. Pastore*}

Department of Physics, University of York, Heslington, York, Y010 5DD, UK

\section{J. Navarro}

IFIC (CSIC-Universidad de Valencia), Apartado Postal 22085, E-46.071-Valencia, Spain

\begin{abstract}
We present the partial wave decomposition of the pure neutron matter equation of state based on the extended Skyrme functional N3LO. This decomposition is particularly useful to extract detailed informations on tensor properties in infinite medium. The results of this paper confirm those obtained in the case of infinite symmetric matter.
\end{abstract}

The 26th International Nuclear Physics Conference

11-16 September, 2016

Adelaide, Australia

${ }^{*}$ Speaker. 


\section{Introduction}

The Skyrme N3LO pseudo-potential [1,2] is an extension of the standard Skyrme interaction [3], obtained by including all possible combinations of momentum operators up to 6th order. In a recent series of articles $[4,5,6,7,8]$, we have studied the properties of the Skyrme N3LO gauge-invariant interaction (see $[1,2]$ ) in infinite nuclear matter. Our main result is that the inclusion of higher order gradients gives an excellent flexibility, allowing us to give a fair description of the main physical results obtained with more involved calculations such as Brueckner-HartreeFock (BHF) $[9,10]$ based on the Argonne AV14 two-body force plus the Urbana VII three-body force. In particular, we have shown in Ref. [5] that including higher order gradients allows to reproduce with very high accuracy the spin-isospin decomposition of the Equation of State (EoS) for infinite symmetric nuclear matter. It is important to point out that this result can not be achieved by employing a standard Skyrme interaction [11] even with some extra density dependent terms. The BHF calculations also provide the different partial waves of the EoS. We have thus performed such a decomposition for the N3LO pseudo-potential [8] and compared to the BHF results. We have clearly obtained a very good agreement and observed that the main contributions to the EoS come from $S, P, D$ and marginally from $F$ waves: the contribution of higher order waves is negligible for values of the density close to the saturation point. As a consequence, we argued that the N3LO pseudo-potential contains the main physical terms necessary to properly reproduce the physics around saturation density. It is worth recalling that in his original article, Skyrme [3] already pointed out that the interaction should also include $D$ wave terms, i.e. 4-th power in gradients.

In Ref [8], we have considered two families of finite-range interactions: the Gogny [12] and Yukawa [13] interactions. Although a finite-range interaction contains by definition all partial waves, a systematic analysis of the properties of the EoS [8] revealed that the only relevant contributions to the EoS come from $S, P, D$ waves and to a lesser extent from $F$ waves. This implies that N3LO can constitute a good approximation to any finite-range interaction. In the present article, we want to complete our studies on the infinite matter properties of the Skyrme N3LO pseudopotential, by considering the extreme case of isospin asymmetry, i.e. pure neutron matter (PNM). The article is organised as follows: in Sec. 2 we briefly present the formalism to derive the EoS with an effective interaction and its partial wave decomposition for Skyrme N3LO in PNM. In Sec.3, we show how to isolate the contribution of tensor terms and finally in Sec. 4 we give our conclusions.

\section{Equation of state and its decomposition}

The energy per particle in PNM within the Hartree-Fock approximation is given by

$$
\frac{E}{N}=\frac{3}{5} \frac{\hbar^{2} k_{F}^{2}}{2 m}+\frac{1}{2 N} \sum_{i j}\left\langle i j\left|V_{S k}\right| i j-j i\right\rangle
$$

where $k_{F}=\left(3 \pi^{2} \rho_{n}\right)^{1 / 3}$ is the Fermi momentum and $\rho_{n}$ is the density of the system. In the above expression, the antisymmetric matrix elements of the Skyrme N3LO pseudo-potential $V_{S k}$ have to be calculated on plane wave basis and the indices $i, j$ span the occupied states in the Fermi sphere. 
The complete expression for $V_{S k}$ has been given in Ref. [8] in a Cartesian basis. The total energy per particle then reads

$$
\begin{aligned}
\frac{E}{N}= & \frac{3}{5} \frac{\hbar^{2}}{2 m} b^{2} \rho_{n}{ }^{2 / 3}+\frac{1}{4}\left(1-x_{0}^{(0)}\right) t_{0}^{(0)} \rho_{n}+\frac{1}{24}\left(1-x_{3}\right) t_{3} \rho_{n}{ }^{\alpha+1} \\
& +\frac{3}{80} b^{2} C^{(2)} \rho_{n}^{5 / 3}+\frac{9}{280} b^{4} C^{(4)} \rho_{n}^{7 / 3}+\frac{2}{15} b^{6} C^{(6)} \rho_{n}^{3},
\end{aligned}
$$

where we used $b=\left(3 \pi^{2}\right)^{1 / 3}$ and $C^{(n)}=2 t_{1}^{(n)}\left(1-x_{1}^{(n)}\right)+6 t_{2}^{(n)}\left(1+x_{2}^{(n)}\right)$.

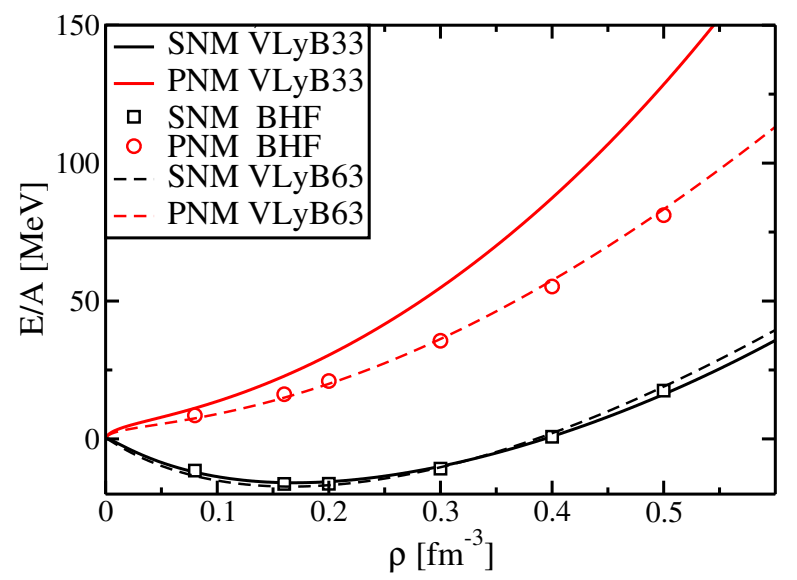

Figure 1: (Color online) Equation of state in SNM and PNM for VLyB33, VLyB63 and BHF. See text for details.

We recall briefly at this point that some parametrisations for N3LO have been obtained in Ref. [5] by fitting the spin-isospin channels of the SNM equation of state on BHF calculations (the PNM results can thus be considered as a prediction). The resulting parametrisations correspond to two popular choices of the power of the density dependent term $\alpha=1 / 3$ for VLyB33 and $\alpha=1 / 6$ for VLyB63. Such a term is needed to obtain both saturation properties and acceptable values of the effective mass [5]. As a consequence the transformation from SNM to PNM is not straightforward and one should pay some extra attention to this term. In Fig. 1, we compare the results of the VLyB33 and VLyB63 parametrisations against BHF results for SNM and PNM. We see that the trend is correct for VLyB33 but the agreement is very good for VLyB63.

\subsection{Partial wave decomposition}

Following the procedure highlighted in Ref. [4], it is possible to project the HF basis onto a basis with total angular momentum $\vec{J}=\vec{L}+\vec{S}$, where $\vec{L}$ is the total angular momentum and $\vec{S}$ is the total spin. The interaction part of the EoS can be thus written as

$$
V=\sum_{J L S} V\left({ }^{2 S+1} L_{J}\right)
$$


where we used the standard spectroscopic notation ${ }^{2 S+1} L_{J}$ for a partial wave with quantum numbers (S,L,J). At variance with the SNM case described in Refs. [4, 8], the isospin quantum number $T$ is no longer relevant in the case of PNM so that the selection rules are different. A close inspection leads to the fact that only spin-singlet $(S=0)$ components for even values of $L$ and spin-triplet ( $S=1$ ) states for odd values of $L$ can appear. We finally obtain the following expressions

$$
\begin{aligned}
V\left({ }^{1} S_{0}\right) & =\frac{1}{4} t_{0}\left(1-x_{0}\right) \rho_{n}+\frac{1}{24} t_{3}\left(1-x_{3}\right) \rho_{n}^{\alpha+1}+\frac{3}{40} t_{1}\left(1-x_{1}\right) \rho_{n} k_{F_{n}}^{2} \\
& +\frac{3}{70} t_{1}^{(4)}\left(1-x_{1}^{(4)}\right) \rho_{n} k_{F_{n}}^{4}+\frac{2}{15} t_{1}^{(6)}\left(1-x_{1}^{(6)}\right) \rho_{n} k_{F_{n}}^{6} \\
V\left({ }^{3} P_{0}\right) & =\frac{1}{40} t_{2}\left(1+x_{2}\right) \rho_{n} k_{F_{n}}^{2}+\frac{3}{140} t_{2}^{(4)}\left(1+x_{2}^{(4)}\right) \rho_{n} k_{F_{n}}^{4}+\frac{2}{25} t_{2}^{(6)}\left(1+x_{2}^{(6)}\right) \rho_{n} k_{F_{n}}^{6} \\
& -\frac{1}{20} t_{o} \rho_{n} k_{F_{n}}^{2}-\frac{3}{25} t_{o}^{(4)} \rho_{n} k_{F_{n}}^{4}-\frac{6}{125} t_{o}^{(6)} \rho_{n} k_{F_{n}}^{6}+\frac{1}{30} W_{0} \rho_{n} k_{F_{n}}^{2}, \\
V\left({ }^{3} P_{1}\right) & =\frac{3}{40} t_{2}\left(1+x_{2}\right) \rho_{n} k_{F_{n}}^{2}+\frac{9}{140} t_{2}^{(4)}\left(1+x_{2}^{(4)}\right) \rho_{n} k_{F_{n}}^{4}+\frac{6}{25} t_{2}^{(6)}\left(1+x_{2}^{(6)}\right) \rho_{n} k_{F_{n}}^{6} \\
& +\frac{3}{40} t_{o} \rho_{n} k_{F_{n}}^{2}+\frac{9}{50} t_{o}^{(4)} \rho_{n} k_{F_{n}}^{4}+\frac{9}{125} t_{o}^{(6)} \rho_{n} k_{F_{n}}^{6}+\frac{1}{20} W_{0} \rho_{n} k_{F_{n}}^{2}, \\
V\left({ }^{3} P_{2}\right) & =\frac{1}{8} t_{2}\left(1+x_{2}\right) \rho_{n} k_{F_{n}}^{2}+\frac{3}{28} t_{2}^{(4)}\left(1+x_{2}^{(4)}\right) \rho_{n} k_{F_{n}}^{4}+\frac{2}{5} t_{2}^{(6)}\left(1+x_{2}^{(6)}\right) \rho_{n} k_{F_{n}}^{6} \\
& -\frac{1}{40} t_{o} \rho_{n} k_{F_{n}}^{2}-\frac{3}{50} t_{o}^{(4)} \rho_{n} k_{F_{n}}^{4}-\frac{3}{125} t_{o}^{(6)} \rho_{n} k_{F_{n}}^{6}-\frac{1}{12} W_{0} \rho_{n} k_{F_{n}}^{2}, \\
V\left({ }^{1} D_{2}\right) & =\frac{3}{140} t_{1}^{(4)}\left(1-x_{1}^{(4)}\right) \rho_{n} k_{F_{n}}^{4}+\frac{2}{15} t_{1}^{(6)}\left(1-x_{2}^{(6)}\right) \rho_{n} k_{F_{n}}^{6} \\
V\left({ }^{3} F_{2}\right) & =\frac{2}{105} t_{2}^{(6)}\left(1+x_{2}^{(6)}\right) \rho_{n} k_{F_{n}}^{6}-\frac{4}{875} t_{o}^{(6)} \rho_{n} k_{F_{n}}^{6}, \\
V\left({ }^{3} F_{3}\right) & =\frac{2}{75} t_{2}^{(6)}\left(1+x_{2}^{(6)}\right) \rho_{n} k_{F_{n}}^{6}+\frac{1}{125} t_{o}^{(6)} \rho_{n} k_{F_{n}}^{6} \\
V\left({ }^{3} F_{4}\right) & =\frac{6}{175} t_{2}^{(6)}\left(1+x_{2}^{(6)}\right) \rho_{n} k_{F_{n}}^{6}-\frac{3}{875} t_{o}^{(6)} \rho_{n} k_{F_{n}}^{6} .
\end{aligned}
$$

We see in the above expressions that spin-orbit and tensor terms appear explicitly in the different partial waves but cancel exactly, as it should be, when the spin channels or the total EoS Eq.2.2 are reconstructed. These two terms give rise to a splitting of partial waves for a given value of orbital angular momentum $L$.

In Fig. 2, we compare the above partial wave decomposition of the EoS obtained using VLyB33 and VLyB63 with the BHF results. As already discussed in Ref. [6], some partial waves are coupled (such as ${ }^{3} P_{2}-{ }^{3} F_{2}$ by instance) in the BHF calculation. At HF level, such a coupling do not appear so that we simply sum the two independent contributions. The agreement is quite good for the VLyB33 interaction, while it deteriorates for the VLyB63 one. It is worth noticing that in both cases, we do get the correct trend and magnitude for all the partial waves.

\section{Tensor properties of Skyrme N3LO in PNM}

We clearly see in the partial wave expansion that, apart from a trivial J-degeneracy factor, the central term of the two-body interaction is the same for a given value of $L$. As a consequence, 

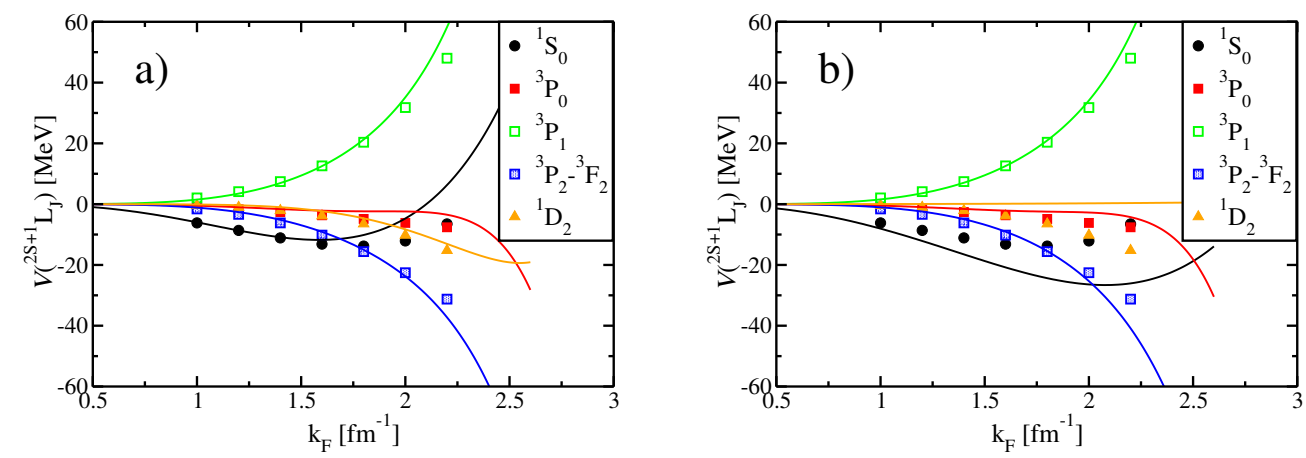

Figure 2: (Color online) We compare the partial wave $V\left({ }^{2 S+1} L_{J}\right)$ decomposition of the EoS: the dots represent the BHF results while the solid lines represent the results obtained with VLyB33 (panel a) and VLyB63 (panel b). See text for details

we can take $a d$ - hoc combinations of partial waves to cancel out the contribution of the central term and thus isolate the tensor and spin-orbit components (a similar procedure has already been discussed in Ref. [7]). By inspecting the equations, we find that the relevant combinations are

$$
\begin{gathered}
\delta_{P}=\frac{1}{3} V\left({ }^{3} P_{1}\right)-V\left({ }^{3} P_{0}\right), \\
\delta_{F}=\frac{1}{7} V\left({ }^{3} F_{3}\right)-\frac{1}{9} V\left({ }^{3} F_{4}\right) .
\end{gathered}
$$

It is worth noticing that while this procedure is exact at HF level, this is not the case for BHF calculations, since the $a b$-initio terms enter in the determination of the different components in a more complicated way. In Fig. 3 we compare the partial wave differences given in Eqs. 3.13.2 using VLyB33 only (VLyB63 and VLyB33 have the same tensor and spin-orbit terms) with the BHF results. We observe that both channels are very well reproduced, both in magnitude and trend. Despite such very good results, we stress again as in Ref. [5] that the values we got here for the tensor and spin-orbit terms seem to be a factor of $2 / 3$ stronger than the standard values usually obtained in finite nuclei.

Finally, we have also calculated the Landau parameters and compare them with those obtained by Chiral Effective Field Theory $(\chi$-EFT). Following Refs. [14, 15], the Landau parameters for the tensor terms read

$$
\begin{aligned}
& N_{0}^{-1} H_{0}=\frac{1}{8} k_{F_{n}}^{2} L_{2}^{n}[h]+\frac{1}{2} k_{F_{n}}^{4} L_{4}^{n}[h]+\frac{1}{3} k_{F_{n}}^{6} L_{6}^{n}[h] \\
& N_{0}^{-1} H_{1}=-\frac{1}{2} k_{F_{n}}^{4} L_{4}^{n}[h]-\frac{1}{2} k_{F_{n}}^{6} L_{6}^{n}[h] \\
& N_{0}^{-1} H_{2}=\frac{1}{6} k_{F_{n}}^{6} L_{6}^{n}[h]
\end{aligned}
$$

where $N_{0}=m_{n}^{*} k_{F_{n}} / \pi^{2}$ is the density of states at the Fermi surface and $L_{(x)}^{n}[h]=4 t_{o}^{(x)}$. As discussed in Ref. [16], the spin-orbit term does not contribute to the Landau parameters. 


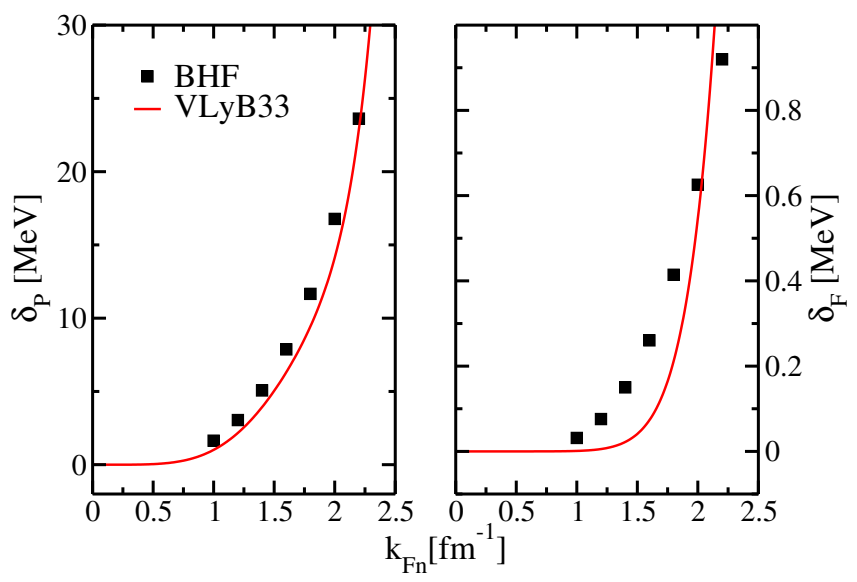

Figure 3: (Color online) Comparing the partial wave differences as given in Eqs. 3.1-3.2 using VLyB33 and the original BHF results. See text for details.

In Fig. 4 , we show the evolution of the tensor Landau parameters $H_{l}$ obtained with VLyB33 as a function of the density together with the $\chi$-EFT results obtained with cut off regulator $\Lambda=450$ $\mathrm{MeV}[17,18]$. It is important to recall that in $a b$-initio methods there are extra tensor parameters related to the center of mass motion $K_{l}$ and $L_{l}$. In Ref. [19], we have examined these Landau parameters and we have observed that they are of the same order of magnitude of $H_{l}$. On Fig.4, we thus have to focus on trends only. The only other noticeable aspect in this figure is that we can see that the Landau parameters of VLyB33 are much stronger than the ones obtained from $\chi$-EFT. This goes in the same direction as the anomaly already spotted in Ref. [5] about the excessive magnitude of tensor parameters in infinite matter.

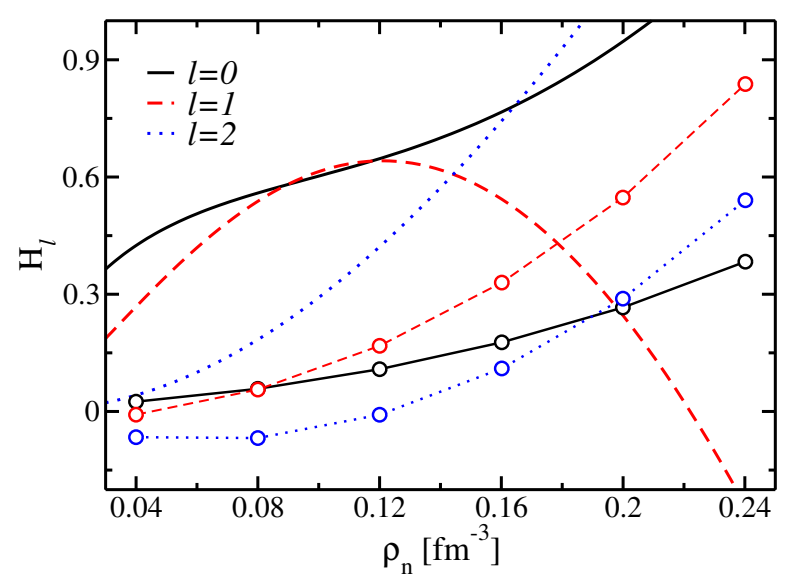

Figure 4: (Color online) Evolution of the tensor Landau parameters $H_{l}$ obtained with VLyB33 as a function of the density of the system. The dots represent the results obtained from $\chi$-EFT [18]. See text for details. 


\section{Conclusions}

In this paper, we have studied the properties of the Skyrme N3LO pseudo-potential in the case of pure neutron matter by performing a partial wave expansion of the equation of state. This allowed us to isolate the tensor and spin-orbit contributions. At present time, the only few existing parametrisations of the N3LO pseudo-potential have been derived using properties of infinite symmetric matter [5, 6]. We have used two sets, named VLyB33 and VLyB63, that have a different exponent in the density dependent term. Although PNM does not appear into their fitting protocol, they give a very fair reproduction of the total EoS and its partial wave decomposition, showing the consistency of this approach. Concerning the tensor and spin-orbit contributions, the agreement between VLyB and Brueckner-Hartree-Fock is very good. Finally, we have also calculated the tensor Landau parameters and compared them with recent $\chi$-EFT results. One indication from this comparison is that the tensor parameters extracted with SNM and compatible with PNM are twice as big as one should expect from a proper fit including also finite-nuclei properties. However, as already discussed in the conclusion of Ref. [5], given the large uncertainties on both the sign and the magnitude of the Skyrme tensor term [20], our approach can be considered as a valuable starting point for a complete fit of the interaction.

\section{Acknowledgments}

We thank M. Baldo for providing us with the BHF results. The work of J.N. has been supported by grant FIS2014-51948-C2-1-P, Mineco (Spain).

\section{References}

[1] B. G. Carlsson, J. Dobaczewski and M. KortelainenPhys. Rev. C 78 (2008) 044326.

[2] F. Raimondi, B. G. Carlsson and J. Dobaczewski, Effective pseudopotential for energy density functionals with higher-order derivatives, Phys. Rev. C 83 (May, 2011) 054311.

[3] T. H. R. Skyrme, The effective nuclear potential, Nucl. Phys. 9 (1959) 615.

[4] D. Davesne, J. Meyer, A. Pastore and J. Navarro, Partial wave decomposition of the n3lo equation of state, Phys. Scr. 90 (2015) 114002.

[5] D. Davesne, J. Navarro, P. Becker, R. Jodon, J. Meyer and A. Pastore, Extended skyrme pseudopotential deduced from infinite nuclear matter properties, Phys. Rev. C 91 (Jun, 2015) 064303.

[6] D. Davesne, A. Pastore and J. Navarro, Extended skyrme equation of state in asymmetric nuclear matter, Astronomy \& Astrophysics 585 (2016) A83.

[7] D. Davesne, P. Becker, A. Pastore and J. Navarro, Partial-wave decomposition of the finite-range effective tensor interaction, Physical Review C 93 (2016) 064001.

[8] D. Davesne, P. Becker, A. Pastore and J. Navarro, Infinite matter properties and zero-range limit of non-relativistic finite-range interactions, Annals of Physics 375 (2016) 288-312.

[9] M. Baldo, I. Bombaci, L. Ferreira, G. Giansiracusa and U. Lombardo, Nuclear matter within the continuous choice, Physical Review C $\mathbf{4 3}$ (1991) 2605. 
[10] M. Baldo, I. Bombaci and G. Burgio, Microscopic nuclear equation of state with three-body forces and neutron star structure, Astron. Astrophys. 328 (1997) 274-282.

[11] T. Lesinski, K. Bennaceur, T. Duguet and J. Meyer, Isovector splitting of nucleon effective masses, ab initio benchmarks and extended stability criteria for skyrme energy functionals, Phys. Rev. C 74 (Oct, 2006) 044315.

[12] J. Dechargé and D. Gogny, Hartree-fock-bogolyubov calculations with the d 1 effective interaction on spherical nuclei, Phys. Rev. C 21 (1980) 1568.

[13] H. Nakada, Hartree-fock approach to nuclear matter and finite nuclei with m3y-type nucleon-nucleon interactions, Phys. Rev. C 68 (2003) 014316.

[14] D. Davesne, A. Pastore and J. Navarro, Fitting n3lo pseudo-potentials through central plus tensor landau parameters, Journal of Physics G: Nuclear and Particle Physics 41 (2014) 065104.

[15] A. Pastore, D. Davesne and J. Navarro, Linear response of homogeneous nuclear matter with energy density functionals, Phys. Reports 563 (2015) 1-67.

[16] A. Pastore, D. Davesne and J. Navarro, Nuclear matter response function with a central plus tensor landau interaction, J. Phys. G: Nucl. Part. Phys. 41 (2014) 055103.

[17] J. W. Holt, N. Kaiser and W. Weise, Chiral fermi liquid approach to neutron matter, Phys. Rev. C 87 (Jan, 2013) 014338.

[18] L. Coraggio, J. Holt, N. Itaco, R. Machleidt and F. Sammarruca, Reduced regulator dependence of neutron-matter predictions with perturbative chiral interactions, Physical Review C 87 (2013) 014322.

[19] D. Davesne, J. W. Holt, A. Pastore and J. Navarro, Effect of three-body forces on response functions in infinite neutron matter, Physical Review C 91 (2015) 014323.

[20] T. Lesinski, M. Bender, K. Bennaceur, T. Duguet and J. Meyer, Tensor part of the skyrme energy density functional: Spherical nuclei, Phys. Rev. C 76 (Jul, 2007) 014312. 